\title{
On Semiprime Gamma Near-Rings with Perpendicular Generalized 3-Derivations
}

\author{
Ikram A. Saed \\ Department of Applied Sciences \\ University of Technology, Baghdad, Iraq . \\ Email : ikramsaed1962@gmail.com
}

Recived : $11 \backslash 2 \backslash 2019$

Revised : $21 \backslash 2 \backslash 2019$

Accepted : 27\2\2019

Available online : $23 / 4 / 2019$

\begin{abstract}
:
In this paper, we introduce the notion of perpendicular generalized 3-derivations in semiprime gamma near-rings and present several necessary and sufficient conditions for generalized 3-derivations on semiprime gamma near-rings to be perpendicular .
\end{abstract}

KeyWords: Semiprime $\Gamma$-near-ring, 3-derivations, Generalized 3-derivations , perpendicular 3derivations, perpendicular generalized 3-derivations .

Mathematics Subject Classification: 16A70, 16N60, 16W25 . 


\section{Introduction}

This paper consists of two sections. In section one, we recall some known definitions and necessary lemmas that we will use it later in this paper. In section two, we begin by introducing definition of perpendicular generalized 3-derivations in $\Gamma$-near-rings. Furthermore, several conditions are given to make the two generalized 3-derivations perpendicular.

\section{Basic Concept}

\section{Definition 1.1:[2]}

A right near-ring ( resp. a left near-ring ) is a nonempty set $\mathrm{N}$ equipped with two binary operations + and . such that

(i) $(\mathrm{N},+)$ is a group (not necessarily abelian )

(ii) $(\mathrm{N}$, . ) is a semigroup

(iii) For $x, y, z \in N$, we have

$(x+y) z=x z+y z(\operatorname{resp} . z(x+y)=z x+z y)$

Example $1.2:[2]$

Let $\mathrm{G}$ be a group ( not necessarily abelian ) then all mapping of $\mathrm{G}$ into itself form a right near-ring $\mathrm{M}(\mathrm{G})$ with regard to point wise addition and multiplication by composite .

\section{Definition 1.3:[2]}

Let $M$ and $\Gamma$ be additive abelian groups. If there exists a mapping $\mathrm{M} \times \Gamma \times \mathrm{M} \rightarrow \mathrm{M}$ : (a , $\alpha, \mathrm{b}) \rightarrow \mathrm{a} \alpha \mathrm{b}$ which satisfies the conditions : for every $\mathrm{a}, \mathrm{b}, \mathrm{c} \in \mathrm{M}, \alpha, \beta \in \Gamma$

(i) $(\mathrm{a}+\mathrm{b}) \alpha \mathrm{c}=\mathrm{a} \alpha \mathrm{c}+\mathrm{b} \alpha \mathrm{c}$

$\mathrm{a}(\alpha+\beta) \mathrm{b}=\mathrm{a} \alpha \mathrm{b}+\mathrm{a} \beta \mathrm{b}$

$\mathrm{a} \alpha(\mathrm{b}+\mathrm{c})=\mathrm{a} \alpha \mathrm{b}+\mathrm{a} \alpha \mathrm{c}$

(ii) ( a $\alpha \mathrm{b}) \beta \mathrm{c}=\mathrm{a} \alpha(\mathrm{b} \beta \mathrm{c})$

Then $M$ is called a $\Gamma$-ring .

Example 1.4 :[2]

Let $\mathrm{R}$ be a ring, the additive abelian groups $\mathrm{M}=\mathrm{M}_{2 \times 3}(\mathrm{R})$ and $\Gamma=\mathrm{M}_{3 \times 2}(\mathrm{R})$ denotes the sets of all $2 \times 3$ matrices over $R$ and $3 \times 2$ matrices over $\mathrm{R}$ respectively. Then $\mathrm{M}$ is $\Gamma$-ring.

\section{Definition 1.5:[5]}

A $\Gamma$-near-ring is a triple $(\mathrm{N},+, \Gamma)$ where (i) $(\mathrm{N},+)$ is a group ( not necessarily abelian ) (ii) $\Gamma$ is a non-empty set of binary operations on $\mathrm{N}$ such that for each $\alpha \in \Gamma,(\mathrm{N},+, \alpha)$ is a left near-ring .

(iii) $\mathrm{x} \alpha(\mathrm{y} \beta \mathrm{z})=(\mathrm{x} \alpha \mathrm{y}) \beta \mathrm{z}$ for all $\mathrm{x}, \mathrm{y}, \mathrm{z} \in \mathrm{N}, \alpha$, $\beta \in \Gamma$.

\section{Definition 1.6:[5]}

Let $\mathrm{W}$ be a $\Gamma$-near-ring, the set $\mathrm{W}_{0}=\{\mathrm{x} \in \mathrm{W}$ : $0 \alpha \mathrm{x}=0, \alpha \in \Gamma\}$ is said to be zero-symmetric part of $\mathrm{W}$. A $\Gamma$-near-ring $\mathrm{W}$ is called zerosymmetric if $\mathrm{W}=\mathrm{W}_{0}$.

\section{Definition 1.7:[5]}

A $\Gamma$-near-ring $\mathrm{W}$ is said to be a prime $\Gamma$ near-ring when $\mathrm{W}$ satisfy the following for $\mathrm{a}$, $\mathrm{b} \in \mathrm{W}, \mathrm{a} \Gamma \mathrm{W} \Gamma \mathrm{b}=\{0\}$ implies $\mathrm{a}=0$ or $\mathrm{b}=0$

\section{Definition 1.8: [5]}

A $\Gamma$-near-ring $\mathrm{W}$ is said to be a semiprime when $\mathrm{W}$ satisfy the following for $\mathrm{a} \in \mathrm{W}$, $\mathrm{a} \Gamma \mathrm{W} \Gamma \mathrm{a}=\{0\}$ implies $\mathrm{a}=0$.

\section{Definition 1.9:[5]}

A $\Gamma$-near-ring $\mathrm{W}$ is said to be 2-torsion free if for all $\mathrm{x} \in \mathrm{W}, 2 \mathrm{x}=0$ implies $\mathrm{x}=0$.

\section{Definition 1.10:[3]}

Let $\mathrm{W}$ be a $\Gamma$-near-ring . An additive map $\mathrm{T}$ : $\mathrm{W} \rightarrow \mathrm{W}$ is said to be a derivation if $\mathrm{T}(\mathrm{a} \alpha \mathrm{b})$ $=\mathrm{T}(\mathrm{a}) \alpha \mathrm{b}+\mathrm{a} \alpha \mathrm{T}(\mathrm{b})$ for every a , b $\in \mathrm{W}, \alpha \in \Gamma$.

\section{Definition 1.11:[3]}

Let $\mathrm{W}$ be a $\Gamma$-near-ring and $\mathrm{D}: \mathrm{W} \rightarrow \mathrm{W}$ be an additive map. If there exists a derivation $\mathrm{d}: \mathrm{W}$ $\rightarrow \mathrm{W}$ such that $\mathrm{D}(\mathrm{x} \alpha \mathrm{y})=\mathrm{D}(\mathrm{x}) \alpha \mathrm{y}+\mathrm{x} \alpha \mathrm{d}(\mathrm{y})$ holds for all $\mathrm{x}, \mathrm{y} \in \mathrm{W}, \alpha \in \Gamma$, then $\mathrm{D}$ is called a generalized derivation.

\section{Definition 1.12:[1]}

Suppose that $\mathrm{W}$ is a near-ring . An 3-additive mapping $\mathrm{d}: \mathrm{W} \times \mathrm{W} \times \mathrm{W} \rightarrow \mathrm{W}$ is called 3derivation if the relations :

$\mathrm{d}\left(\mathrm{s}_{1} \mathrm{~s}_{1}{ }^{\prime}, \mathrm{s}_{2}, \mathrm{~s}_{3}\right)=\mathrm{d}\left(\mathrm{s}_{1}, \mathrm{~s}_{2}, \mathrm{~s}_{3}\right) \mathrm{s}_{1}{ }^{\prime}+\mathrm{s}_{1} \mathrm{~d}\left(\mathrm{~s}_{1}{ }^{\prime}, \mathrm{s}_{2}, \mathrm{~s}_{3}\right)$ $\mathrm{d}\left(\mathrm{s}_{1}, \mathrm{~s}_{2} \mathrm{~s}_{2}^{\prime}, \mathrm{s}_{3}\right)=\mathrm{d}\left(\mathrm{s}_{1}, \mathrm{~s}_{2}, \mathrm{~s}_{3}\right) \mathrm{s}_{2}^{\prime}+\mathrm{s}_{2} \mathrm{~d}\left(\mathrm{~s}_{1}, \mathrm{~s}_{2}^{\prime}, \mathrm{s}_{3}\right)$ $\mathrm{d}\left(\mathrm{s}_{1}, \mathrm{~s}_{2}, \mathrm{~s}_{3} \mathrm{~s}_{3}{ }^{\prime}\right)=\mathrm{d}\left(\mathrm{s}_{1}, \mathrm{~s}_{2}, \mathrm{~s}_{3}\right) \mathrm{s}_{3}{ }^{\prime}+\mathrm{s}_{3} \mathrm{~d}\left(\mathrm{~s}_{1}, \mathrm{~s}_{2}, \mathrm{~s}_{3}{ }^{\prime}\right)$ hold for all $s_{1}, s_{1}^{\prime}, s_{2}, s_{2}^{\prime}, s_{3}, s_{3}{ }^{\prime} \in W$.

Example $1.13:[1]$

Let $\mathrm{S}$ be a commutative near-ring .

Let us define 
$\mathrm{W}=\left\{\left(\begin{array}{ll}r & u \\ 0 & 0\end{array}\right): r, u, 0 \in S\right\}$.

And $\mathrm{d}: \mathrm{WxWx} \mathrm{W} \rightarrow \mathrm{W}$

$\mathrm{d}\left(\left(\begin{array}{cc}r_{1} & u_{1} \\ 0 & 0\end{array}\right),\left(\begin{array}{cc}r_{2} & u_{2} \\ 0 & 0\end{array}\right),\left(\begin{array}{cc}r_{3} & u_{3} \\ 0 & 0\end{array}\right)\right)=$

$\left(\begin{array}{cc}0 & r_{1} r_{2} r_{3} \\ 0 & 0\end{array}\right)$

Then $\mathrm{d}$ is 3-derivation of $\mathrm{W}$.

\section{Definition 1.14:[1]}

Suppose that $\mathrm{W}$ is a near-ring and $\mathrm{d}$ be 3 derivation of $W$. An 3-additive mapping $\mathrm{f}$ : $\mathrm{WxWxW} \rightarrow \mathrm{W}$ is said to be generalized 3derivation of $\mathrm{W}$ associated with $\mathrm{d}$ if the relations

$\mathrm{f}\left(\mathrm{s}_{1} \mathrm{~s}_{1}^{\prime}, \mathrm{s}_{2}, \mathrm{~s}_{3}\right)=\mathrm{f}\left(\mathrm{s}_{1}, \mathrm{~s}_{2}, \mathrm{~s}_{3}\right) \mathrm{s}_{1}^{\prime}+\mathrm{s}_{1} \mathrm{~d}\left(\mathrm{~s}_{1}^{\prime}, \mathrm{s}_{2}, \mathrm{~s}_{3}\right)$ $\mathrm{f}\left(\mathrm{s}_{1}, \mathrm{~s}_{2} \mathrm{~s}_{2}{ }^{\prime}, \mathrm{s}_{3}\right)=\mathrm{f}\left(\mathrm{s}_{1}, \mathrm{~s}_{2}, \mathrm{~s}_{3}\right) \mathrm{s}_{2}{ }^{\prime}+\mathrm{s}_{2} \mathrm{~d}\left(\mathrm{~s}_{1}, \mathrm{~s}_{2}{ }^{\prime}, \mathrm{s}_{3}\right)$ $\mathrm{f}\left(\mathrm{s}_{1}, \mathrm{~s}_{2}, \mathrm{~s}_{3} \mathrm{~s}_{3}^{\prime}\right)=\mathrm{f}\left(\mathrm{s}_{1}, \mathrm{~s}_{2}, \mathrm{~s}_{3}\right) \mathrm{s}_{3}{ }^{\prime}+\mathrm{s}_{3} \mathrm{~d}\left(\mathrm{~s}_{1}, \mathrm{~s}_{2}, \mathrm{~s}_{3}{ }^{\prime}\right)$ hold for all $\mathrm{s}_{1}, \mathrm{~s}_{1}^{\prime}, \mathrm{s}_{2}, \mathrm{~s}_{2}^{\prime}, \mathrm{s}_{3}, \mathrm{~s}_{3}^{\prime} \in \mathrm{W}$.

\section{Example $1.15:[1]$}

Let $\mathrm{S}$ be a commutative near-ring .

Let us define

$\mathrm{W}=\left\{\left(\begin{array}{ll}0 & r \\ 0 & u\end{array}\right): r, u, 0 \in S\right\}$.

And d, f : WxWxW $\rightarrow \mathrm{W}$,

$\mathrm{d}\left(\left(\begin{array}{ll}0 & r_{1} \\ 0 & u_{1}\end{array}\right),\left(\begin{array}{ll}0 & r_{2} \\ 0 & u_{2}\end{array}\right),\left(\begin{array}{ll}0 & r_{3} \\ 0 & u_{3}\end{array}\right)\right)=$

$\left(\begin{array}{cc}0 & r_{1} r_{2} r_{3} \\ 0 & 0\end{array}\right)$

$\mathrm{f}\left(\left(\begin{array}{cc}0 & r_{1} \\ 0 & u_{1}\end{array}\right),\left(\begin{array}{ll}0 & r_{2} \\ 0 & u_{2}\end{array}\right),\left(\begin{array}{ll}0 & r_{3} \\ 0 & u_{3}\end{array}\right)\right)=$

$\left(\begin{array}{cc}0 & 0 \\ 0 & u_{1} \\ u_{2} u_{3}\end{array}\right)$

Then $\mathrm{f}$ is a generalized 3-derivation of $\mathrm{W}$.

\section{Lemma $1.16:[4]$}

Let $\mathrm{W}$ be a 2 -torsion free semiprime $\Gamma$-nearring and $\mathrm{a}, \mathrm{b} \in \mathrm{W}$. When

(i) $\mathrm{a} \alpha \mathrm{x} \beta \mathrm{b}=0$ for all $\mathrm{x} \in \mathrm{W}$ and $\alpha, \beta \in \Gamma$.

(ii) $\mathrm{b} \alpha \mathrm{x} \beta \mathrm{a}=0$ for all $\mathrm{x} \in \mathrm{W}$ and $\alpha, \beta \in \Gamma$.

(iii) $\mathrm{a} \alpha \mathrm{x} \beta \mathrm{b}+\mathrm{b} \alpha \mathrm{x} \beta \mathrm{a}=0$ for all $\mathrm{x} \in \mathrm{W}$ and $\alpha$, $\beta \in \Gamma$. are equivalent

When one of the above is satisfied, implies a $\Gamma$ $\mathrm{b}=\mathrm{b} \Gamma \mathrm{a}=0$.

\section{Lemma $1.17:[4]$}

Assume that $\mathrm{W}$ is a $\Gamma$-near-ring and $\mathrm{D}$ be a generalized derivation of $\mathrm{W}$. When the next conditions are satisfied : (i) $(\mathrm{D}(\mathrm{x}) \alpha \mathrm{y}+\mathrm{x} \alpha \mathrm{d}(\mathrm{y})) \beta \mathrm{z}=\mathrm{D}(\mathrm{x}) \alpha \mathrm{y} \beta \mathrm{z}+$ $\mathrm{x} \alpha \mathrm{d}(\mathrm{y}) \beta \mathrm{z}$

For every $\mathrm{x}, \mathrm{y}, \mathrm{z} \in \mathrm{W}$ and $\alpha, \beta \in \Gamma$.

(ii) $(\mathrm{d}(\mathrm{x}) \alpha \mathrm{y}+\mathrm{x} \alpha \mathrm{d}(\mathrm{y})) \beta \mathrm{z}=\mathrm{d}(\mathrm{x}) \alpha \mathrm{y} \beta \mathrm{z}+\mathrm{x} \alpha \mathrm{d}(\mathrm{y})$

$\beta \mathrm{z}$

for every $\mathrm{x}, \mathrm{y}, \mathrm{z} \in \mathrm{W}, \alpha, \beta \in \Gamma$.

\section{Perpendicular Generalized 3- \\ Derivations}

First we introduce the basic definition in this paper

\section{Definition 2.1:}

Let $\mathrm{W}$ be a $\Gamma$-near-ring and $\mathrm{P}, \mathrm{Q}$ be two generalized 3-derivations of $\mathrm{W} . \mathrm{P}$ and $\mathrm{Q}$ are called perpendicular if next relation $\mathrm{P}\left(\mathrm{s}_{1}, \mathrm{~s}_{2}, \mathrm{~s}_{3}\right) \Gamma \mathrm{W} \Gamma \mathrm{Q}\left(\mathrm{n}_{1}, \mathrm{n}_{2}, \mathrm{n}_{3}\right)=0=\mathrm{Q}\left(\mathrm{n}_{1}\right.$, $\left.\mathrm{n}_{2}, \mathrm{n}_{3}\right) \Gamma \mathrm{W} \Gamma \mathrm{P}\left(\mathrm{s}_{1}, \mathrm{~s}_{2}, \mathrm{~s}_{3}\right)$ holds for every $\mathrm{s}_{1}, \mathrm{~s}_{2}, \mathrm{~s}_{3}, \mathrm{n}_{1}, \mathrm{n}_{2}, \mathrm{n}_{3} \in \mathrm{W}$.

\section{Lemma 2.2:}

Assume that $\mathrm{W}$ is a 2-torsion free semiprime $\Gamma$-near-ring and $\mathrm{P}, \mathrm{Q}$ are two generalized 3derivations of $\mathrm{W}$ with associated 3derivations $\mathrm{p}$ and $\mathrm{q}$ of $\mathrm{W}$ respectively. When $\mathrm{p}$ and $\mathrm{q}$ are perpendicular, so next conditions are satisfied :

(i) $\mathrm{P}\left(\mathrm{s}_{1}, \mathrm{~s}_{2}, \mathrm{~s}_{3}\right) \Gamma \mathrm{Q}\left(\mathrm{n}_{1}, \mathrm{n}_{2}, \mathrm{n}_{3}\right)=\mathrm{Q}\left(\mathrm{s}_{1}, \mathrm{~s}_{2}, \mathrm{~s}_{3}\right) \Gamma$ $\mathrm{P}\left(\mathrm{n}_{1}, \mathrm{n}_{2}, \mathrm{n}_{3}\right)=0$

For every $\mathrm{s}_{1}, \mathrm{~s}_{2}, \mathrm{~s}_{3}, \mathrm{n}_{1}, \mathrm{n}_{2}, \mathrm{n}_{3} \in \mathrm{W}$.

(ii)p and $q$ are perpendicular and $\mathrm{p}\left(\mathrm{s}_{1}, \mathrm{~s}_{2}, \mathrm{~s}_{3}\right) \Gamma \mathrm{Q}\left(\mathrm{n}_{1}, \mathrm{n}_{2}, \mathrm{n}_{3}\right)=\mathrm{Q}\left(\mathrm{n}_{1}, \mathrm{n}_{2}, \mathrm{n}_{3}\right) \Gamma$ $\mathrm{p}\left(\mathrm{s}_{1}, \mathrm{~s}_{2}, \mathrm{~s}_{3}\right)=0$

for every $s_{1}, s_{2}, s_{3}, n_{1}, n_{2}, n_{3} \in W$.

(iii) $q$ and $P$ are perpendicular and $q\left(s_{1}, s_{2}, s_{3}\right) \Gamma$ $\mathrm{P}\left(\mathrm{n}_{1}, \mathrm{n}_{2}, \mathrm{n}_{3}\right)=\mathrm{P}\left(\mathrm{n}_{1}, \mathrm{n}_{2}, \mathrm{n}_{3}\right) \Gamma \mathrm{q}\left(\mathrm{s}_{1}, \mathrm{~s}_{2}, \mathrm{~s}_{3}\right)=0$

for every $s_{1}, s_{2}, s_{3}, n_{1}, n_{2}, n_{3} \in W$.

(iv)p and $q$ are perpendicular and $\mathrm{p}\left(\mathrm{q}\left(\mathrm{s}_{1}, \mathrm{~s}_{2}, \mathrm{~s}_{3}\right), \mathrm{n}_{2}, \mathrm{n}_{3}\right)=0$ for every $\mathrm{s}_{1}, \mathrm{~s}_{2}, \mathrm{~s}_{3}$ $, \mathrm{n}_{2}, \mathrm{n}_{3} \in \mathrm{W}$

(v) $\mathrm{p}\left(\mathrm{Q}\left(\mathrm{s}_{1}, \mathrm{~s}_{2}, \mathrm{~s}_{3}\right), \mathrm{n}_{2}, \mathrm{n}_{3}\right)=\mathrm{Q}\left(\mathrm{p}\left(\mathrm{s}_{1}, \mathrm{~s}_{2}, \mathrm{~s}_{3}\right)\right.$, $\left.\mathrm{n}_{2}, \mathrm{n}_{3}\right)=0$

for all $\mathrm{s}_{1}, \mathrm{~s}_{2}, \mathrm{~s}_{3}, \mathrm{n}_{2}, \mathrm{n}_{3} \in \mathrm{W}$, and $\mathrm{q}\left(\mathrm{P}\left(\mathrm{s}_{1}, \mathrm{~s}_{2}, \mathrm{~s}_{3}\right), \mathrm{n}_{2}, \mathrm{n}_{3}\right)=\mathrm{P}\left(\mathrm{q}\left(\mathrm{s}_{1}, \mathrm{~s}_{2}, \mathrm{~s}_{3}\right), \mathrm{n}_{2}, \mathrm{n}_{3}\right.$ )$=0$

for all $\mathrm{s}_{1}, \mathrm{~s}_{2}, \mathrm{~s}_{3}, \mathrm{n}_{2}, \mathrm{n}_{3} \in \mathrm{W}$. 
(vi) $\mathrm{Q}\left(\mathrm{P}\left(\mathrm{s}_{1}, \mathrm{~s}_{2}, \mathrm{~s}_{3}\right), \mathrm{n}_{2}, \mathrm{n}_{3}\right)=\mathrm{P}\left(\mathrm{Q}\left(\mathrm{s}_{1}, \mathrm{~s}_{2}, \mathrm{~s}_{3}\right)\right.$, $\left.\mathrm{n}_{2}, \mathrm{n}_{3}\right)=0$

for all $s_{1}, s_{2}, s_{3}, n_{2}, n_{3} \in W$.

\section{Proof:}

(i)Since $P$ and $Q$ are perpendicular, implies that

$\mathrm{P}\left(\mathrm{s}_{1}, \mathrm{~s}_{2}, \mathrm{~s}_{3}\right) \alpha \mathrm{s} \beta \mathrm{Q}\left(\mathrm{n}_{1}, \mathrm{n}_{2}, \mathrm{n}_{3}\right)=0$ for every $\mathrm{s}, \mathrm{s}_{1}, \mathrm{~s}_{2}, \mathrm{~s}_{3}, \mathrm{n}_{1}, \mathrm{n}_{2}, \mathrm{n}_{3} \in \mathrm{W}$ and $\alpha, \beta \in \Gamma$.

By Lemma 1.17 we get

$\mathrm{P}\left(\mathrm{s}_{1}, \mathrm{~s}_{2}, \mathrm{~s}_{3}\right) \alpha \mathrm{Q}\left(\mathrm{n}_{1}, \mathrm{n}_{2}, \mathrm{n}_{3}\right)=\mathrm{Q}\left(\mathrm{s}_{1}, \mathrm{~s}_{2}, \mathrm{~s}_{3}\right) \alpha$ $\mathrm{P}\left(\mathrm{n}_{1}, \mathrm{n}_{2}, \mathrm{n}_{3}\right)=0 \quad$ for all $\mathrm{s}_{1}, \mathrm{~s}_{2}, \mathrm{~s}_{3}, \mathrm{n}_{1}$, $\mathrm{n}_{2}, \mathrm{n}_{3} \in \mathrm{W}$ and $\alpha \in \Gamma$.

(ii) by (i) we get

$\mathrm{P}\left(\mathrm{s}_{1}, \mathrm{~s}_{2}, \mathrm{~s}_{3}\right) \alpha \mathrm{Q}\left(\mathrm{n}_{1}, \mathrm{n}_{2}, \mathrm{n}_{3}\right)=0$

for every $\mathrm{s}_{1}, \mathrm{~s}_{2}, \mathrm{~s}_{3}, \mathrm{n}_{1}, \mathrm{n}_{2}, \mathrm{n}_{3} \in \mathrm{W}, \alpha \in \Gamma$. Replacing $\mathrm{s}_{1}$ by $\mathrm{s}_{1}^{\prime} \beta \mathrm{s}_{1}$, where $\mathrm{s}_{1}{ }^{\prime} \in \mathrm{W}$ and $\beta$ $\in \Gamma$ in previous equation and using Lemma 1.17 we get

$0=\mathrm{P}\left(\mathrm{s}_{1}^{\prime} \beta \mathrm{s}_{1}, \mathrm{~s}_{2}, \mathrm{~s}_{3}\right) \alpha \mathrm{Q}\left(\mathrm{n}_{1}, \mathrm{n}_{2}, \mathrm{n}_{3}\right)$

$=\left[\mathrm{P}\left(\mathrm{s}_{1}^{\prime}, \mathrm{s}_{2}, \mathrm{~s}_{3}\right) \beta \mathrm{s}_{1}+\mathrm{s}_{1}^{\prime} \beta \mathrm{p}\left(\mathrm{s}_{1}, \mathrm{~s}_{2}, \mathrm{~s}_{3}\right)\right] \alpha$

$\mathrm{Q}\left(\mathrm{n}_{1}, \mathrm{n}_{2}, \mathrm{n}_{3}\right)$

$=\mathrm{P}\left(\mathrm{s}_{1}{ }^{\prime}, \mathrm{s}_{2}, \mathrm{~s}_{3}\right) \beta \mathrm{s}_{1} \alpha \mathrm{Q}\left(\mathrm{n}_{1}, \mathrm{n}_{2}, \mathrm{n}_{3}\right)$

$+\mathrm{s}_{1}^{\prime} \beta \mathrm{p}\left(\mathrm{s}_{1}, \mathrm{~s}_{2}, \mathrm{~s}_{3}\right) \alpha \mathrm{Q}\left(\mathrm{n}_{1}, \mathrm{n}_{2}, \mathrm{n}_{3}\right)$

$=\mathrm{s}_{1}^{\prime} \beta \mathrm{p}\left(\mathrm{s}_{1}, \mathrm{~s}_{2}, \mathrm{~s}_{3}\right) \alpha \mathrm{Q}\left(\mathrm{n}_{1}, \mathrm{n}_{2}, \mathrm{n}_{3}\right)$

for all $\mathrm{s}_{1}^{\prime}, \mathrm{s}_{1}, \mathrm{~s}_{2}, \mathrm{~s}_{3}, \mathrm{n}_{1}, \mathrm{n}_{2}, \mathrm{n}_{3} \in \mathrm{W}$ and $\alpha, \beta$ $\in \Gamma$.

Since $\mathrm{W}$ is semiprime we get

$\mathrm{p}\left(\mathrm{s}_{1}, \mathrm{~s}_{2}, \mathrm{~s}_{3}\right) \alpha \mathrm{Q}\left(\mathrm{n}_{1}, \mathrm{n}_{2}, \mathrm{n}_{3}\right)=0$

for every $\mathrm{s}_{1}, \mathrm{~s}_{2}, \mathrm{~s}_{3}, \mathrm{n}_{1}, \mathrm{n}_{2}, \mathrm{n}_{3} \in \mathrm{W}$ and $\alpha \in$ $\Gamma$.

Now replacing $\mathrm{s}_{1}$ by $\mathrm{s}_{1} \beta \mathrm{s}_{1}^{\prime}$, where $\mathrm{s}_{1}^{\prime} \in \mathrm{W}$ and $\beta \in \Gamma$ in previous equation and using Lemma 1.17 (ii), we get

$0=\mathrm{p}\left(\mathrm{s}_{1} \beta \mathrm{s}_{1}^{\prime}, \mathrm{s}_{2}, \mathrm{~s}_{3}\right) \alpha \mathrm{Q}\left(\mathrm{n}_{1}, \mathrm{n}_{2}, \mathrm{n}_{3}\right)$

$=\left[\mathrm{p}\left(\mathrm{s}_{1}, \mathrm{~s}_{2}, \mathrm{~s}_{3}\right) \beta \mathrm{s}_{1}{ }^{\prime}+\mathrm{s}_{1} \beta \mathrm{p}\left(\mathrm{s}_{1}{ }^{\prime}, \mathrm{s}_{2}, \mathrm{~s}_{3}\right)\right] \alpha$

$\mathrm{Q}\left(\mathrm{n}_{1}, \mathrm{n}_{2}, \mathrm{n}_{3}\right)$

$=\mathrm{p}\left(\mathrm{s}_{1}, \mathrm{~s}_{2}, \mathrm{~s}_{3}\right) \beta \mathrm{s}_{1}{ }^{\prime} \alpha \mathrm{Q}\left(\mathrm{n}_{1}, \mathrm{n}_{2}, \mathrm{n}_{3}\right)$

$+\mathrm{s}_{1} \beta \mathrm{p}\left(\mathrm{s}_{1}^{\prime}, \mathrm{s}_{2}, \mathrm{~s}_{3}\right) \alpha \mathrm{Q}\left(\mathrm{n}_{1}, \mathrm{n}_{2}, \mathrm{n}_{3}\right)$

$=\mathrm{p}\left(\mathrm{s}_{1}, \mathrm{~s}_{2}, \mathrm{~s}_{3}\right) \beta \mathrm{s}_{1}{ }^{\prime} \alpha \mathrm{Q}\left(\mathrm{n}_{1}, \mathrm{n}_{2}, \mathrm{n}_{3}\right)$

for all $\mathrm{s}_{1}^{\prime}, \mathrm{s}_{1}, \mathrm{~s}_{2}, \mathrm{~s}_{3}, \mathrm{n}_{1}, \mathrm{n}_{2}, \mathrm{n}_{3} \in \mathrm{W}, \alpha, \beta \in \Gamma$

By Lemma 1.16 we obtain

$\mathrm{p}\left(\mathrm{s}_{1}, \mathrm{~s}_{2}, \mathrm{~s}_{3}\right) \Gamma \mathrm{Q}\left(\mathrm{n}_{1}, \mathrm{n}_{2}, \mathrm{n}_{3}\right)=\mathrm{Q}\left(\mathrm{n}_{1}, \mathrm{n}_{2}, \mathrm{n}_{3}\right) \Gamma$

$\mathrm{p}\left(\mathrm{s}_{1}, \mathrm{~s}_{2}, \mathrm{~s}_{3}\right)=0$

for all $\mathrm{s}_{1}, \mathrm{~s}_{2}, \mathrm{~s}_{3}, \mathrm{n}_{1}, \mathrm{n}_{2}, \mathrm{n}_{3} \in \mathrm{W}$. (iii) The proof is similar to (ii)

(iv) By (i) we get

$\mathrm{P}\left(\mathrm{s}_{1}, \mathrm{~s}_{2}, \mathrm{~s}_{3}\right) \alpha \mathrm{Q}\left(\mathrm{n}_{1}, \mathrm{n}_{2}, \mathrm{n}_{3}\right)=0$

for all $\mathrm{s}_{1}, \mathrm{~s}_{2}, \mathrm{~s}_{3}, \mathrm{n}_{1}, \mathrm{n}_{2}, \mathrm{n}_{3} \in \mathrm{W}, \alpha \in \Gamma$.

Replacing $\mathrm{s}_{1}$ by $\mathrm{s}_{1}^{\prime} \beta \mathrm{s}_{1}$, where $\mathrm{s}_{1}{ }^{\prime} \in \mathrm{W}$ and $\beta$

$\in \Gamma$ in previous equation and using Lemma

1.17 we get

$0=\mathrm{P}\left(\mathrm{s}_{1}^{\prime} \beta \mathrm{s}_{1}, \mathrm{~s}_{2}, \mathrm{~s}_{3}\right) \alpha \mathrm{Q}\left(\mathrm{n}_{1}, \mathrm{n}_{2}, \mathrm{n}_{3}\right)$

$=\left[\mathrm{P}\left(\mathrm{s}_{1}^{\prime}, \mathrm{s}_{2}, \mathrm{~s}_{3}\right) \beta \mathrm{s}_{1}+\mathrm{s}_{1}^{\prime} \beta \mathrm{p}\left(\mathrm{s}_{1}, \mathrm{~s}_{2}, \mathrm{~s}_{3}\right)\right] \alpha$

$\mathrm{Q}\left(\mathrm{n}_{1}, \mathrm{n}_{2}, \mathrm{n}_{3}\right)$

$=\mathrm{P}\left(\mathrm{s}_{1}^{\prime}, \mathrm{s}_{2}, \mathrm{~s}_{3}\right) \beta \mathrm{s}_{1} \alpha \mathrm{Q}\left(\mathrm{n}_{1}, \mathrm{n}_{2}, \mathrm{n}_{3}\right)$

$+\mathrm{s}_{1}^{\prime} \beta \mathrm{p}\left(\mathrm{s}_{1}, \mathrm{~s}_{2}, \mathrm{~s}_{3}\right) \alpha \mathrm{Q}\left(\mathrm{n}_{1}, \mathrm{n}_{2}, \mathrm{n}_{3}\right)$

$=\mathrm{s}_{1}^{\prime} \beta \mathrm{p}\left(\mathrm{s}_{1}, \mathrm{~s}_{2}, \mathrm{~s}_{3}\right) \alpha \mathrm{Q}\left(\mathrm{n}_{1}, \mathrm{n}_{2}, \mathrm{n}_{3}\right)$

For every $\mathrm{s}_{1}^{\prime}, \mathrm{s}_{1}, \mathrm{~s}_{2}, \mathrm{~s}_{3}, \mathrm{n}_{1}, \mathrm{n}_{2}, \mathrm{n}_{3} \in \mathrm{W}, \alpha, \beta$ $\in \Gamma$.

Replacing $\mathrm{n}_{1}$ by $\mathrm{n}_{1} \delta \mathrm{n}_{1}^{\prime}$, where $\mathrm{n}_{1}^{\prime} \in \mathrm{W}$ and $\delta \in \Gamma$ in previous equation and using (ii), we get

$0=\mathrm{s}_{1}^{\prime} \beta \mathrm{p}\left(\mathrm{s}_{1}, \mathrm{~s}_{2}, \mathrm{~s}_{3}\right) \alpha \mathrm{Q}\left(\mathrm{n}_{1} \delta \mathrm{n}_{1}^{\prime}, \mathrm{n}_{2}, \mathrm{n}_{3}\right)$

$=\mathrm{s}_{1}^{\prime} \beta \mathrm{p}\left(\mathrm{s}_{1}, \mathrm{~s}_{2}, \mathrm{~s}_{3}\right) \alpha\left[\mathrm{Q}\left(\mathrm{n}_{1}, \mathrm{n}_{2}, \mathrm{n}_{3}\right) \delta \mathrm{n}_{1}^{\prime}+\mathrm{n}_{1} \delta\right.$

$\mathrm{q}\left(\mathrm{n}_{1}^{\prime}, \mathrm{n}_{2}, \mathrm{n}_{3}\right)$

$=\mathrm{s}_{1}^{\prime} \beta \mathrm{p}\left(\mathrm{s}_{1}, \mathrm{~s}_{2}, \mathrm{~s}_{3}\right) \alpha \mathrm{Q}\left(\mathrm{n}_{1}, \mathrm{n}_{2}, \mathrm{n}_{3}\right) \delta \mathrm{n}_{1}^{\prime}$

$+\mathrm{s}_{1}^{\prime} \beta \mathrm{p}\left(\mathrm{s}_{1}, \mathrm{~s}_{2}, \mathrm{~s}_{3}\right) \alpha \mathrm{n}_{1} \delta \mathrm{q}\left(\mathrm{n}_{1}^{\prime}, \mathrm{n}_{2}, \mathrm{n}_{3}\right)$

$=\mathrm{s}_{1}^{\prime} \beta \mathrm{p}\left(\mathrm{s}_{1}, \mathrm{~s}_{2}, \mathrm{~s}_{3}\right) \alpha \mathrm{n}_{1} \delta \mathrm{q}\left(\mathrm{n}_{1}^{\prime}, \mathrm{n}_{2}, \mathrm{n}_{3}\right)$

for all $\mathrm{s}_{1}^{\prime}, \mathrm{s}_{1}, \mathrm{~s}_{2}, \mathrm{~s}_{3}, \mathrm{n}_{1}, \mathrm{n}_{1}^{\prime}, \mathrm{n}_{2}, \mathrm{n}_{3} \in \mathrm{W}, \alpha$,

$\beta, \delta \in \Gamma$.

Semiprimeness of $\mathrm{W}$ implies

$\mathrm{p}\left(\mathrm{s}_{1}, \mathrm{~s}_{2}, \mathrm{~s}_{3}\right) \alpha \mathrm{n}_{1} \delta \mathrm{q}\left(\mathrm{n}_{1}^{\prime}, \mathrm{n}_{2}, \mathrm{n}_{3}\right)=0$

for every $\mathrm{s}_{1}, \mathrm{~s}_{2}, \mathrm{~s}_{3}, \mathrm{n}_{1}, \mathrm{n}_{1}^{\prime}, \mathrm{n}_{2}, \mathrm{n}_{3} \in \mathrm{W}, \alpha, \delta$ $\in \Gamma$.

That is $\mathrm{p}$ and $\mathrm{q}$ are perpendicular, then we have

$0=\mathrm{p}\left(\mathrm{p}\left(\mathrm{v}_{1}, \mathrm{v}_{2}, \mathrm{v}_{3}\right) \alpha \mathrm{n}_{1} \beta \mathrm{q}\left(\mathrm{s}_{1}, \mathrm{~s}_{2}, \mathrm{~s}_{3}\right), \mathrm{n}_{2}, \mathrm{n}_{3}\right)$

$=\mathrm{p}\left(\mathrm{p}\left(\mathrm{v}_{1}, \mathrm{v}_{2}, \mathrm{v}_{3}\right), \mathrm{n}_{2}, \mathrm{n}_{3}\right) \alpha \mathrm{n}_{1} \beta \mathrm{q}\left(\mathrm{s}_{1}, \mathrm{~s}_{2}, \mathrm{~s}_{3}\right)$

$+\mathrm{p}\left(\mathrm{v}_{1}, \mathrm{v}_{2}, \mathrm{v}_{3}\right) \alpha \mathrm{p}\left(\mathrm{n}_{1} \beta \mathrm{q}\left(\mathrm{s}_{1}, \mathrm{~s}_{2}, \mathrm{~s}_{3}\right), \mathrm{n}_{2}, \mathrm{n}_{3}\right)$

for all $\mathrm{s}_{1}, \mathrm{~s}_{2}, \mathrm{~s}_{3}, \mathrm{n}_{1}, \mathrm{n}_{2}, \mathrm{n}_{3}, \mathrm{v}_{1}, \mathrm{v}_{2}, \mathrm{v}_{3} \in \mathrm{W}, \alpha$ , $\beta \in \Gamma$.

Since $\mathrm{p}, \mathrm{q}$ are perpendicular, we have

$0=\mathrm{p}\left(\mathrm{v}_{1}, \mathrm{v}_{2}, \mathrm{v}_{3}\right) \alpha \mathrm{p}\left(\mathrm{n}_{1} \beta \mathrm{q}\left(\mathrm{s}_{1}, \mathrm{~s}_{2}, \mathrm{~s}_{3}\right), \mathrm{n}_{2}, \mathrm{n}_{3}\right)$

$=\mathrm{p}\left(\mathrm{v}_{1}, \mathrm{v}_{2}, \mathrm{v}_{3}\right) \alpha \mathrm{p}\left(\mathrm{n}_{1}, \mathrm{n}_{2}, \mathrm{n}_{3}\right) \beta \mathrm{q}\left(\mathrm{s}_{1}, \mathrm{~s}_{2}, \mathrm{~s}_{3}\right)$

$+\mathrm{p}\left(\mathrm{v}_{1}, \mathrm{v}_{2}, \mathrm{v}_{3}\right) \alpha \mathrm{n}_{1} \beta \mathrm{p}\left(\mathrm{q}\left(\mathrm{s}_{1}, \mathrm{~s}_{2}, \mathrm{~s}_{3}\right), \mathrm{n}_{2}, \mathrm{n}_{3}\right)$

Since $\mathrm{p}, \mathrm{q}$ are perpendicular, we conclude $\mathrm{p}\left(\mathrm{v}_{1}, \mathrm{v}_{2}, \mathrm{v}_{3}\right) \alpha \mathrm{n}_{1} \beta \mathrm{p}\left(\mathrm{q}\left(\mathrm{s}_{1}, \mathrm{~s}_{2}, \mathrm{~s}_{3}\right), \mathrm{n}_{2}, \mathrm{n}_{3}\right)=0$ for all $\mathrm{s}_{1}, \mathrm{~s}_{2}, \mathrm{~s}_{3}, \mathrm{n}_{1}, \mathrm{n}_{2}, \mathrm{n}_{3}, \mathrm{v}_{1}, \mathrm{v}_{2}, \mathrm{v}_{3} \in \mathrm{W}$, $\alpha, \beta \in \Gamma$. 
Replacing $\mathrm{v}_{1}$ by $\mathrm{q}\left(\mathrm{s}_{1}, \mathrm{~s}_{2}, \mathrm{~s}_{3}\right)$ and $\mathrm{v}_{2}$ by $\mathrm{n}_{2}, \mathrm{v}_{3}$ by $\mathrm{n}_{3}$, implies

$\mathrm{p}\left(\mathrm{q}\left(\mathrm{s}_{1}, \mathrm{~s}_{2}, \mathrm{~s}_{3}\right), \mathrm{n}_{2}, \mathrm{n}_{3}\right) \Gamma \mathrm{W} \Gamma \mathrm{p}\left(\mathrm{q}\left(\mathrm{s}_{1}, \mathrm{~s}_{2}, \mathrm{~s}_{3}\right), \mathrm{n}_{2}\right.$ , $\left.\mathrm{n}_{3}\right)=\{0\}$

for all $\mathrm{s}_{1}, \mathrm{~s}_{2}, \mathrm{~s}_{3}, \mathrm{n}_{2}, \mathrm{n}_{3} \in \mathrm{W}$. Semipimeness of $\mathrm{W}$ implies that

$\mathrm{p}\left(\mathrm{q}\left(\mathrm{s}_{1}, \mathrm{~s}_{2}, \mathrm{~s}_{3}\right), \mathrm{n}_{2}, \mathrm{n}_{3}\right)=0$ for all $\mathrm{s}_{1}, \mathrm{~s}_{2}, \mathrm{~s}_{3}, \mathrm{n}_{2}$ , $\mathrm{n}_{3} \in \mathrm{W}$.

Now we prove part 5 and part 6

Using part 2 and part 4 , we have

$0=\mathrm{Q}\left(\mathrm{p}\left(\mathrm{s}_{1}, \mathrm{~s}_{2}, \mathrm{~s}_{3}\right) \alpha \mathrm{s} \beta \mathrm{Q}\left(\mathrm{n}_{1}, \mathrm{n}_{2}, \mathrm{n}_{3}\right), \mathrm{n}_{2}, \mathrm{n}_{3}\right)$

$=\mathrm{Q}\left(\mathrm{p}\left(\mathrm{s}_{1}, \mathrm{~s}_{2}, \mathrm{~s}_{3}\right), \mathrm{n}_{2}, \mathrm{n}_{3}\right) \alpha \mathrm{s} \beta \mathrm{Q}\left(\mathrm{n}_{1}, \mathrm{n}_{2}, \mathrm{n}_{3}\right)$

$+\mathrm{p}\left(\mathrm{s}_{1}, \mathrm{~s}_{2}, \mathrm{~s}_{3}\right) \alpha \mathrm{q}\left(s \beta \mathrm{Q}\left(\mathrm{n}_{1}, \mathrm{n}_{2}, \mathrm{n}_{3}\right), \mathrm{n}_{2}, \mathrm{n}_{3}\right)$

$=\mathrm{Q}\left(\mathrm{p}\left(\mathrm{s}_{1}, \mathrm{~s}_{2}, \mathrm{~s}_{3}\right), \mathrm{n}_{2}, \mathrm{n}_{3}\right) \alpha \mathrm{s} \beta \mathrm{Q}\left(\mathrm{n}_{1}, \mathrm{n}_{2}, \mathrm{n}_{3}\right)$

for all $\mathrm{s}, \mathrm{s}_{1}, \mathrm{~s}_{2}, \mathrm{~s}_{3}, \mathrm{n}_{1}, \mathrm{n}_{2}, \mathrm{n}_{3} \in \mathrm{W}, \alpha, \beta \in \Gamma$

Replacing $\mathrm{n}_{1}$ by $\mathrm{p}\left(\mathrm{s}_{1}, \mathrm{~s}_{2}, \mathrm{~s}_{3}\right)$, we get

$\mathrm{Q}\left(\mathrm{p}\left(\mathrm{s}_{1}, \mathrm{~s}_{2}, \mathrm{~s}_{3}\right), \mathrm{n}_{2}, \mathrm{n}_{3}\right) \alpha \mathrm{W} \beta \mathrm{Q}\left(\mathrm{p}\left(\mathrm{s}_{1}, \mathrm{~s}_{2}, \mathrm{~s}_{3}\right)\right.$, $\left.\mathrm{n}_{2}, \mathrm{n}_{3}\right)=\{0\}$

Semiprimeness of $\mathrm{W}$ implies that

$\mathrm{Q}\left(\mathrm{p}\left(\mathrm{s}_{1}, \mathrm{~s}_{2}, \mathrm{~s}_{3}\right), \mathrm{n}_{2}, \mathrm{n}_{3}\right)=0$ for all $\mathrm{s}_{1}, \mathrm{~s}_{2}, \mathrm{~s}_{3}$, $\mathrm{n}_{2}, \mathrm{n}_{3} \in \mathrm{W}$.

Similarly, we see that since

$\mathrm{p}\left(\mathrm{Q}\left(\mathrm{s}_{1}, \mathrm{~s}_{2}, \mathrm{~s}_{3}\right) \alpha \mathrm{s} \beta \mathrm{p}\left(\mathrm{n}_{1}, \mathrm{n}_{2}, \mathrm{n}_{3}\right), \mathrm{n}_{2}, \mathrm{n}_{3}\right)=0$

$\mathrm{P}\left(\mathrm{q}\left(\mathrm{s}_{1}, \mathrm{~s}_{2}, \mathrm{~s}_{3}\right) \alpha \mathrm{s} \beta \mathrm{P}\left(\mathrm{n}_{1}, \mathrm{n}_{2}, \mathrm{n}_{3}\right), \mathrm{n}_{2}, \mathrm{n}_{3}\right)=0$

$\mathrm{q}\left(\mathrm{P}\left(\mathrm{s}_{1}, \mathrm{~s}_{2}, \mathrm{~s}_{3}\right) \alpha \mathrm{s} \beta \mathrm{q}\left(\mathrm{n}_{1}, \mathrm{n}_{2}, \mathrm{n}_{3}\right), \mathrm{n}_{2}, \mathrm{n}_{3}\right)=0$

$\mathrm{P}\left(\mathrm{Q}\left(\mathrm{s}_{1}, \mathrm{~s}_{2}, \mathrm{~s}_{3}\right) \alpha \mathrm{s} \beta \mathrm{P}\left(\mathrm{n}_{1}, \mathrm{n}_{2}, \mathrm{n}_{3}\right), \mathrm{n}_{2}, \mathrm{n}_{3}\right)=0$

$\mathrm{Q}\left(\mathrm{P}\left(\mathrm{s}_{1}, \mathrm{~s}_{2}, \mathrm{~s}_{3}\right) \alpha \mathrm{s} \beta \mathrm{Q}\left(\mathrm{n}_{1}, \mathrm{n}_{2}, \mathrm{n}_{3}\right), \mathrm{n}_{2}, \mathrm{n}_{3}\right)=0$

Hold for every $\mathrm{s}, \mathrm{s}_{1}, \mathrm{~s}_{2}, \mathrm{~s}_{3}, \mathrm{n}_{1}, \mathrm{n}_{2}, \mathrm{n}_{3} \in \mathrm{W}$,

$\alpha, \beta \in \Gamma$,

we get

$\mathrm{p}\left(\mathrm{Q}\left(\mathrm{s}_{1}, \mathrm{~s}_{2}, \mathrm{~s}_{3}\right), \mathrm{n}_{2}, \mathrm{n}_{3}\right)=0$

$\mathrm{P}\left(\mathrm{q}\left(\mathrm{s}_{1}, \mathrm{~s}_{2}, \mathrm{~s}_{3}\right), \mathrm{n}_{2}, \mathrm{n}_{3}\right)=0$

$\mathrm{q}\left(\mathrm{P}\left(\mathrm{s}_{1}, \mathrm{~s}_{2}, \mathrm{~s}_{3}\right), \mathrm{n}_{2}, \mathrm{n}_{3}\right)=0$

$\mathrm{P}\left(\mathrm{Q}\left(\mathrm{s}_{1}, \mathrm{~s}_{2}, \mathrm{~s}_{3}\right), \mathrm{n}_{2}, \mathrm{n}_{3}\right)=0$ and

$\mathrm{Q}\left(\mathrm{P}\left(\mathrm{s}_{1}, \mathrm{~s}_{2}, \mathrm{~s}_{3}\right), \mathrm{n}_{2}, \mathrm{n}_{3}\right)=0$

for all $s_{1}, s_{2}, s_{3}, n_{2}, n_{3} \in W$.

\section{Theorem 2.3 :}

Assume that $\mathrm{W}$ is a 2-torsion free semiprime $\Gamma$-near-ring and $\mathrm{P}, \mathrm{Q}$ are two generalized 3derivations of $\mathrm{W}$ with associated 3-derivations $\mathrm{p}$ and $\mathrm{q}$ of $\mathrm{W}$ respectively, then the next conditions are satisfied:

(i)P and Q are perpendicular. (ii) $\mathrm{P}\left(\mathrm{s}_{1}, \mathrm{~s}_{2}, \mathrm{~s}_{3}\right) \Gamma \mathrm{Q}\left(\mathrm{n}_{1}, \mathrm{n}_{2}, \mathrm{n}_{3}\right)=\mathrm{p}\left(\mathrm{s}_{1}, \mathrm{~s}_{2}, \mathrm{~s}_{3}\right.$

)$\Gamma Q\left(n_{1}, n_{2}, n_{3}\right)=0$

for all $\mathrm{s}_{1}, \mathrm{~s}_{2}, \mathrm{~s}_{3}, \mathrm{n}_{1}, \mathrm{n}_{2}, \mathrm{n}_{3} \in \mathrm{W}$.

(iii) $\mathrm{P}\left(\mathrm{s}_{1}, \mathrm{~s}_{2}, \mathrm{~s}_{3}\right) \Gamma \mathrm{Q}\left(\mathrm{n}_{1}, \mathrm{n}_{2}, \mathrm{n}_{3}\right)=\mathrm{p}\left(\mathrm{s}_{1}, \mathrm{~s}_{2}, \mathrm{~s}_{3}\right.$

)$\Gamma \mathrm{q}\left(\mathrm{n}_{1}, \mathrm{n}_{2}, \mathrm{n}_{3}\right)=0$

And $\mathrm{p}\left(\mathrm{Q}\left(\mathrm{s}_{1}, \mathrm{~s}_{2}, \mathrm{~s}_{3}\right), \mathrm{n}_{2}, \mathrm{n}_{3}\right)=\mathrm{p}\left(\mathrm{q}\left(\mathrm{s}_{1}, \mathrm{~s}_{2}, \mathrm{~s}_{3}\right)\right.$

$\left., \mathrm{n}_{2}, \mathrm{n}_{3}\right)=0$

for every $\mathrm{s}_{1}, \mathrm{~s}_{2}, \mathrm{~s}_{3}, \mathrm{n}_{1}, \mathrm{n}_{2}, \mathrm{n}_{3} \in \mathrm{W}$.

(iv) $\mathrm{P}\left(\mathrm{Q}\left(\mathrm{s}_{1} \alpha \mathrm{s}_{1}{ }^{\prime}, \mathrm{s}_{2}, \mathrm{~s}_{3}\right), \mathrm{n}_{2}, \mathrm{n}_{3}\right)=\mathrm{P}\left(\mathrm{Q}\left(\mathrm{s}_{1}, \mathrm{~s}_{2}, \mathrm{~s}_{3}\right.\right.$ )$\left., \mathrm{n}_{2}, \mathrm{n}_{3}\right) \alpha \mathrm{s}_{1}{ }^{\prime}+\mathrm{s}_{1} \alpha \mathrm{p}\left(\mathrm{q}\left(\mathrm{s}_{1}{ }^{\prime}, \mathrm{s}_{2}, \mathrm{~s}_{3}\right), \mathrm{n}_{2}, \mathrm{n}_{3}\right)$ and

$\mathrm{P}\left(\mathrm{s}_{1}, \mathrm{~s}_{2}, \mathrm{~s}_{3}\right) \Gamma \mathrm{Q}\left(\mathrm{n}_{1}, \mathrm{n}_{2}, \mathrm{n}_{3}\right)=0$

for all $\mathrm{s}_{1}, \mathrm{~s}_{1}^{\prime}, \mathrm{s}_{2}, \mathrm{~s}_{3}, \mathrm{n}_{1}, \mathrm{n}_{2}, \mathrm{n}_{3} \in \mathrm{W}$ and $\alpha \in$ $\Gamma$.

\section{Proof :}

(i) $\Rightarrow$ (ii) and (i) $\Rightarrow$ (iii) are proved in Lemma 2.2

(i), (ii), (iv) and (v) .

On the other hand, (i) $\Rightarrow$ (iv) is obtained from Lemma 2.2 (i), (iv) and (vi) . (ii) $\Rightarrow$ (i) we have

$\mathrm{P}\left(\mathrm{s}_{1}, \mathrm{~s}_{2}, \mathrm{~s}_{3}\right) \Gamma \mathrm{Q}\left(\mathrm{n}_{1}, \mathrm{n}_{2}, \mathrm{n}_{3}\right)=\mathrm{p}\left(\mathrm{s}_{1}, \mathrm{~s}_{2}, \mathrm{~s}_{3}\right.$

) $\Gamma \mathrm{Q}\left(\mathrm{n}_{1}, \mathrm{n}_{2}, \mathrm{n}_{3}\right)=0$

for all $\mathrm{s}_{1}, \mathrm{~s}_{2}, \mathrm{~s}_{3}, \mathrm{n}_{1}, \mathrm{n}_{2}, \mathrm{n}_{3} \in \mathrm{W}$.

Now replacing $\mathrm{s}_{1}$ by $\mathrm{s}_{1} \beta \mathrm{s}_{1}{ }^{\prime}$, where $\mathrm{s}_{1}{ }^{\prime} \in \mathrm{W}$ and $\beta \in \Gamma$ in previous equation, we get $\mathrm{P}\left(\mathrm{s}_{1} \beta \mathrm{s}_{1}^{\prime}, \mathrm{s}_{2}, \mathrm{~s}_{3}\right) \Gamma \mathrm{Q}\left(\mathrm{n}_{1}, \mathrm{n}_{2}, \mathrm{n}_{3}\right)=0$ for all $\mathrm{s}_{1}^{\prime}, \mathrm{s}_{1}, \mathrm{~s}_{2}, \mathrm{~s}_{3}, \mathrm{n}_{1}, \mathrm{n}_{2}, \mathrm{n}_{3} \in \mathrm{W}, \beta \in \Gamma$. Using hypothesis and Lemma 1.17 in last equation, we get

$0=\left[\mathrm{P}\left(\mathrm{s}_{1}, \mathrm{~s}_{2}, \mathrm{~s}_{3}\right) \beta \mathrm{s}_{1}{ }^{\prime}+\mathrm{s}_{1} \beta \mathrm{p}\left(\mathrm{s}_{1}{ }^{\prime}, \mathrm{s}_{2}, \mathrm{~s}_{3}\right)\right] \alpha$

$\mathrm{G}\left(\mathrm{n}_{1}, \mathrm{n}_{2}, \mathrm{n}_{3}\right)$

$=\mathrm{P}\left(\mathrm{s}_{1}, \mathrm{~s}_{2}, \mathrm{~s}_{3}\right) \beta \mathrm{s}_{1}^{\prime} \alpha \mathrm{Q}\left(\mathrm{n}_{1}, \mathrm{n}_{2}, \mathrm{n}_{3}\right)$

$+\mathrm{s}_{1} \beta \mathrm{p}\left(\mathrm{s}_{1}^{\prime}, \mathrm{s}_{2}, \mathrm{~s}_{3}\right) \alpha \mathrm{Q}\left(\mathrm{n}_{1}, \mathrm{n}_{2}, \mathrm{n}_{3}\right)$

$=\mathrm{P}\left(\mathrm{s}_{1}, \mathrm{~s}_{2}, \mathrm{~s}_{3}\right) \beta \mathrm{s}_{1}{ }^{\prime} \alpha \mathrm{Q}\left(\mathrm{n}_{1}, \mathrm{n}_{2}, \mathrm{n}_{3}\right)$

for all $\mathrm{s}_{1}^{\prime}, \mathrm{s}_{1}, \mathrm{~s}_{2}, \mathrm{~s}_{3}, \mathrm{n}_{1}, \mathrm{n}_{2}, \mathrm{n}_{3} \in \mathrm{W}, \alpha, \beta \in \Gamma$

Hence Lemma 1.16 obtain the result .

(iii) $\Rightarrow$ (i) we have

$\mathrm{p}\left(\mathrm{Q}\left(\mathrm{s}_{1}, \mathrm{~s}_{2}, \mathrm{~s}_{3}\right), \mathrm{n}_{2}, \mathrm{n}_{3}\right)=\mathrm{p}\left(\mathrm{q}\left(\mathrm{s}_{1}, \mathrm{~s}_{2}, \mathrm{~s}_{3}\right), \mathrm{n}_{2}, \mathrm{n}_{3}\right.$ )$=0$

for all $\mathrm{s}_{1}, \mathrm{~s}_{2}, \mathrm{~s}_{3}, \mathrm{n}_{2}, \mathrm{n}_{3} \in \mathrm{W}$.

Replacing $\mathrm{s}_{1}$ by $\mathrm{s}_{1} \alpha \mathrm{s}_{1}{ }^{\prime}$, where $\mathrm{s}_{1}{ }^{\prime} \in \mathrm{W}$ and

$\alpha \in \Gamma$ in the equation

$\mathrm{p}\left(\mathrm{Q}\left(\mathrm{s}_{1}, \mathrm{~s}_{2}, \mathrm{~s}_{3}\right), \mathrm{n}_{2}, \mathrm{n}_{3}\right)=0$, we get

$0=\mathrm{p}\left(\mathrm{Q}\left(\mathrm{s}_{1} \alpha \mathrm{s}_{1}^{\prime}, \mathrm{s}_{2}, \mathrm{~s}_{3}\right), \mathrm{n}_{2}, \mathrm{n}_{3}\right)$ 
$=\mathrm{p}\left(\mathrm{Q}\left(\mathrm{s}_{1}, \mathrm{~s}_{2}, \mathrm{~s}_{3}\right) \alpha \mathrm{s}_{1}^{\prime}, \mathrm{n}_{2}, \mathrm{n}_{3}\right)+\mathrm{p}\left(\mathrm{s}_{1} \alpha \mathrm{q}\left(\mathrm{s}_{1}^{\prime}\right.\right.$

$\left.\left., \mathrm{s}_{2}, \mathrm{~s}_{3}\right), \mathrm{n}_{2}, \mathrm{n}_{3}\right)$

$=\mathrm{p}\left(\mathrm{Q}\left(\mathrm{s}_{1}, \mathrm{~s}_{2}, \mathrm{~s}_{\mathrm{n}}\right), \mathrm{n}_{2}, \mathrm{n}_{3}\right) \alpha \mathrm{s}_{1}^{\prime}+$

$\mathrm{Q}\left(\mathrm{s}_{1}, \mathrm{~s}_{2}, \mathrm{~s}_{3}\right) \alpha \mathrm{p}\left(\mathrm{s}_{1}^{\prime}, \mathrm{n}_{2}, \mathrm{n}_{3}\right)+$

$\mathrm{p}\left(\mathrm{s}_{1}, \mathrm{n}_{2}, \mathrm{n}_{3}\right) \alpha \mathrm{q}\left(\mathrm{s}_{1}^{\prime}, \mathrm{s}_{2}, \mathrm{~s}_{3}\right)+$

$\mathrm{s}_{1} \alpha \mathrm{p}\left(\mathrm{q}\left(\mathrm{s}_{1}{ }^{\prime}, \mathrm{s}_{2}, \mathrm{~s}_{3}\right), \mathrm{n}_{2}, \mathrm{n}_{3}\right)$

$=\mathrm{Q}\left(\mathrm{s}_{1}, \mathrm{~s}_{2}, \mathrm{~s}_{3}\right) \alpha \mathrm{p}\left(\mathrm{s}_{1}^{\prime}, \mathrm{n}_{2}, \mathrm{n}_{3}\right)$

For every $\mathrm{s}_{1}^{\prime}, \mathrm{s}_{1}, \mathrm{~s}_{2}, \mathrm{~s}_{3}, \mathrm{n}_{2}, \mathrm{n}_{3} \in \mathrm{W}, \alpha \in \Gamma$. Replacing $\mathrm{s}_{1}^{\prime}$ by $\mathrm{s}_{1}^{\prime} \beta \mathrm{v}$, where $\mathrm{v} \in \mathrm{W}$ and $\beta$ $\in \Gamma$ in previous equation and using it again, we get

$0=\mathrm{Q}\left(\mathrm{s}_{1}, \mathrm{~s}_{2}, \mathrm{~s}_{3}\right) \alpha \mathrm{p}\left(\mathrm{s}_{1}^{\prime} \beta \mathrm{v}, \mathrm{n}_{2}, \mathrm{n}_{3}\right)$

$=\mathrm{Q}\left(\mathrm{s}_{1}, \mathrm{~s}_{2}, \mathrm{~s}_{3}\right) \alpha \mathrm{p}\left(\mathrm{s}_{1}{ }^{\prime}, \mathrm{n}_{2}, \mathrm{n}_{3}\right) \beta \mathrm{v}+\mathrm{Q}\left(\mathrm{s}_{1}, \mathrm{~s}_{2}\right.$,

$\left.\mathrm{s}_{3}\right) \alpha \mathrm{s}_{1}^{\prime} \beta \mathrm{p}\left(\mathrm{v}, \mathrm{n}_{2}, \mathrm{n}_{3}\right)$

$=\mathrm{Q}\left(\mathrm{s}_{1}, \mathrm{~s}_{2}, \mathrm{~s}_{3}\right) \alpha \mathrm{s}_{1}^{\prime} \beta \mathrm{p}\left(\mathrm{v}, \mathrm{n}_{2}, \mathrm{n}_{3}\right)$

for every $\mathrm{v}, \mathrm{s}_{1}^{\prime}, \mathrm{s}_{1}, \mathrm{~s}_{2}, \mathrm{~s}_{3}, \mathrm{n}_{2}, \mathrm{n}_{3} \in \mathrm{W}, \alpha, \beta$ $\in \Gamma$.

Hence by Lemma 1.16 , we have

$\mathrm{p}\left(\mathrm{v}, \mathrm{n}_{2}, \mathrm{n}_{3}\right) \alpha \mathrm{Q}\left(\mathrm{s}_{1}, \mathrm{~s}_{2}, \mathrm{~s}_{3}\right)=0$

for every $\mathrm{v}, \mathrm{s}_{1}, \mathrm{~s}_{2}, \mathrm{~s}_{3}, \mathrm{n}_{2}, \mathrm{n}_{3} \in \mathrm{W}, \alpha \in \Gamma$.

Then (i) follows from (ii)

(iv) $\Rightarrow$ (i) by assumption we have

$\mathrm{P}\left(\mathrm{Q}\left(\mathrm{s}_{1} \alpha \mathrm{s}_{1}{ }^{\prime}, \mathrm{s}_{2}, \mathrm{~s}_{3}\right), \mathrm{n}_{2}, \mathrm{n}_{3}\right)=\mathrm{P}\left(\mathrm{Q}\left(\mathrm{s}_{1}, \mathrm{~s}_{2}, \mathrm{~s}_{3}\right)\right.$,

$\left.\mathrm{n}_{2}, \mathrm{n}_{3}\right) \alpha \mathrm{s}_{1}^{\prime}+\mathrm{s}_{1} \alpha \mathrm{p}\left(\mathrm{q}\left(\mathrm{s}_{1}^{\prime}, \mathrm{s}_{2}, \mathrm{~s}_{3}\right), \mathrm{n}_{2}, \mathrm{n}_{3}\right)$

for all $\mathrm{s}_{1}, \mathrm{~s}_{1}^{\prime}, \mathrm{s}_{2}, \mathrm{~s}_{3}, \mathrm{n}_{2}, \mathrm{n}_{3} \in \mathrm{W}, \alpha \in \Gamma$.

And we also obtained

$\mathrm{P}\left(\mathrm{Q}\left(\mathrm{s}_{1} \alpha \mathrm{s}_{1}{ }^{\prime}, \mathrm{s}_{2}, \mathrm{~s}_{3}\right), \mathrm{n}_{2}, \mathrm{n}_{3}\right)$

$=\mathrm{P}\left(\mathrm{Q}\left(\mathrm{s}_{1}, \mathrm{~s}_{2}, \mathrm{~s}_{3}\right) \alpha \mathrm{s}_{1}{ }^{\prime}+\mathrm{s}_{1} \alpha \mathrm{q}\left(\mathrm{s}_{1}{ }^{\prime}, \mathrm{s}_{2}, \mathrm{~s}_{3}\right), \mathrm{n}_{2}\right.$,

$\left.\mathrm{n}_{3}\right)$

$=\mathrm{P}\left(\mathrm{Q}\left(\mathrm{s}_{1}, \mathrm{~s}_{2}, \mathrm{~s}_{3}\right) \alpha \mathrm{s}_{1}{ }^{\prime}, \mathrm{n}_{2}, \mathrm{n}_{3}\right)+\mathrm{P}\left(\mathrm{s}_{1} \alpha \mathrm{q}\left(\mathrm{s}_{1}{ }^{\prime}\right.\right.$

$\left.\left., \mathrm{s}_{2}, \mathrm{~s}_{3}\right), \mathrm{n}_{2}, \mathrm{n}_{3}\right)$

$=\mathrm{P}\left(\mathrm{Q}\left(\mathrm{s}_{1}, \mathrm{~s}_{2}, \mathrm{~s}_{3}\right), \mathrm{n}_{2}, \mathrm{n}_{3}\right) \alpha \mathrm{s}_{1}{ }^{\prime}+\mathrm{Q}\left(\mathrm{s}_{1}, \mathrm{~s}_{2}, \mathrm{~s}_{3}\right.$

) $\alpha \mathrm{p}\left(\mathrm{s}_{1}{ }^{\prime}, \mathrm{n}_{2}, \mathrm{n}_{3}\right)+$

$\mathrm{P}\left(\mathrm{s}_{1}, \mathrm{n}_{2}, \mathrm{n}_{3}\right) \alpha \mathrm{q}\left(\mathrm{s}_{1}{ }^{\prime}, \mathrm{s}_{2}, \mathrm{~s}_{3}\right)+\mathrm{s}_{1} \alpha \mathrm{p}\left(\mathrm{q}\left(\mathrm{s}_{1}{ }^{\prime}, \mathrm{s}_{2}\right.\right.$,

$\left.\mathrm{s}_{3}\right), \mathrm{n}_{2}, \mathrm{n}_{3}$ )

for every $\mathrm{s}_{1}, \mathrm{~s}_{1}^{\prime}, \mathrm{s}_{2}, \mathrm{~s}_{3}, \mathrm{n}_{2}, \mathrm{n}_{3} \in \mathrm{W}, \alpha \in \Gamma$.

Comparing the above two expression of

$\mathrm{P}\left(\mathrm{Q}\left(\mathrm{s}_{1} \alpha \mathrm{s}_{1}{ }^{\prime}, \mathrm{s}_{2}, \mathrm{~s}_{3}\right), \mathrm{n}_{2}, \mathrm{n}_{3}\right)$ we get

$\mathrm{Q}\left(\mathrm{s}_{1}, \mathrm{~s}_{2}, \mathrm{~s}_{3}\right) \alpha \mathrm{p}\left(\mathrm{s}_{1}^{\prime}, \mathrm{n}_{2}, \mathrm{n}_{3}\right)+\mathrm{P}\left(\mathrm{s}_{1}, \mathrm{n}_{2}, \mathrm{n}_{3}\right) \alpha \mathrm{q}$

$\left(\mathrm{s}_{1}{ }^{\prime}, \mathrm{s}_{2}, \mathrm{~s}_{3}\right)=0$

for every $\mathrm{s}_{1}, \mathrm{~s}_{1}{ }^{\prime}, \mathrm{s}_{2}, \mathrm{~s}_{3}, \mathrm{n}_{2}, \mathrm{n}_{3} \in \mathrm{W}, \alpha \in \Gamma$.

Also by assumption we have

$\mathrm{P}\left(\mathrm{s}_{1}, \mathrm{~s}_{2}, \mathrm{~s}_{3}\right) \alpha \mathrm{Q}\left(\mathrm{n}_{1}, \mathrm{n}_{2}, \mathrm{n}_{3}\right)=0$ for all $\mathrm{s}_{1}, \mathrm{~s}_{2}, \mathrm{~s}_{3}, \mathrm{n}_{1}, \mathrm{n}_{2}, \mathrm{n}_{3} \in \mathrm{W}, \alpha \in \Gamma$.

Replacing $\mathrm{n}_{1}$ by $\mathrm{n}_{1} \beta \mathrm{n}_{1}^{\prime}$ in (2.2) and using it again, we get

$0=\mathrm{P}\left(\mathrm{s}_{1}, \mathrm{~s}_{2}, \mathrm{~s}_{3}\right) \alpha \mathrm{Q}\left(\mathrm{n}_{1} \beta \mathrm{n}_{1}^{\prime}, \mathrm{n}_{2}, \mathrm{n}_{3}\right)$

$=\mathrm{P}\left(\mathrm{s}_{1}, \mathrm{~s}_{2}, \mathrm{~s}_{3}\right) \alpha \mathrm{Q}\left(\mathrm{n}_{1}, \mathrm{n}_{2}, \mathrm{n}_{3}\right) \beta \mathrm{n}_{1}^{\prime}+\mathrm{P}\left(\mathrm{s}_{1}, \mathrm{~s}_{2}\right.$,

$\left.\mathrm{s}_{3}\right) \alpha \mathrm{n}_{1} \beta \mathrm{q}\left(\mathrm{n}_{1}^{\prime}, \mathrm{n}_{2}, \mathrm{n}_{3}\right)$

$=\mathrm{P}\left(\mathrm{s}_{1}, \mathrm{~s}_{2}, \mathrm{~s}_{3}\right) \alpha \mathrm{n}_{1} \beta \mathrm{q}\left(\mathrm{n}_{1}^{\prime}, \mathrm{n}_{2}, \mathrm{n}_{3}\right)$

For every $\mathrm{s}_{1}, \mathrm{~s}_{2}, \mathrm{~s}_{3}, \mathrm{n}_{1}, \mathrm{n}_{1}{ }^{\prime}, \mathrm{n}_{2}, \mathrm{n}_{3} \in \mathrm{W}$ and

$\alpha, \beta \in \Gamma$.

Thus it follows from Lemma 1.16 that

$\mathrm{P}\left(\mathrm{s}_{1}, \mathrm{~s}_{2}, \mathrm{~s}_{3}\right) \alpha \mathrm{q}\left(\mathrm{n}_{1}{ }^{\prime}, \mathrm{n}_{2}, \mathrm{n}_{3}\right)=0$

For every $\mathrm{s}_{1}, \mathrm{~s}_{2}, \mathrm{~s}_{3}, \mathrm{n}_{1}^{\prime}, \mathrm{n}_{2}, \mathrm{n}_{3} \in \mathrm{W}$ and $\alpha \in$

$\Gamma$.

Substituting equation (2.3) in the equation (2.1) yields

$\mathrm{Q}\left(\mathrm{s}_{1}, \mathrm{~s}_{2}, \mathrm{~s}_{3}\right) \alpha \mathrm{p}\left(\mathrm{s}_{1}{ }^{\prime}, \mathrm{n}_{2}, \mathrm{n}_{3}\right)=0$

For every $\mathrm{s}_{1}, \mathrm{~s}_{1}^{\prime}, \mathrm{s}_{2}, \mathrm{~s}_{3}, \mathrm{n}_{2}, \mathrm{n}_{3} \in \mathrm{W}, \alpha \in \Gamma$.

Replacing $\mathrm{s}_{1}^{\prime}$ by $\mathrm{s}_{1}^{\prime} \beta \mathrm{v}$, where $\mathrm{v} \in \mathrm{W}$ and $\beta$

$\in \Gamma$ in previous equation and using it again,

we get

$0=\mathrm{Q}\left(\mathrm{s}_{1}, \mathrm{~s}_{2}, \mathrm{~s}_{3}\right) \alpha \mathrm{p}\left(\mathrm{s}_{1}^{\prime} \beta \mathrm{v}, \mathrm{n}_{2}, \mathrm{n}_{3}\right)$

$=\mathrm{Q}\left(\mathrm{s}_{1}, \mathrm{~s}_{2}, \mathrm{~s}_{3}\right) \alpha \mathrm{p}\left(\mathrm{s}_{1}^{\prime}, \mathrm{n}_{2}, \mathrm{n}_{3}\right) \beta \mathrm{v}+\mathrm{Q}\left(\mathrm{s}_{1}, \mathrm{~s}_{2}, \mathrm{~s}_{3}\right.$

) $\alpha \mathrm{s}_{1}^{\prime} \beta \mathrm{p}\left(\mathrm{v}, \mathrm{n}_{2}, \mathrm{n}_{3}\right)$

$=\mathrm{Q}\left(\mathrm{s}_{1}, \mathrm{~s}_{2}, \mathrm{~s}_{3}\right) \alpha \mathrm{s}_{1}^{\prime} \beta \mathrm{p}\left(\mathrm{v}, \mathrm{n}_{2}, \mathrm{n}_{3}\right)$

for every $\mathrm{v}, \mathrm{s}_{1}^{\prime}, \mathrm{s}_{1}, \mathrm{~s}_{2}, \mathrm{~s}_{3}, \mathrm{n}_{2}, \mathrm{n}_{3} \in \mathrm{W}$ and $\alpha$ ,$\beta \in \Gamma$.

Hence by Lemma 1.16 , we have

$\mathrm{p}\left(\mathrm{v}, \mathrm{n}_{2}, \mathrm{n}_{3}\right) \alpha \mathrm{Q}\left(\mathrm{s}_{1}, \mathrm{~s}_{2}, \mathrm{~s}_{3}\right)=0$

for every $\mathrm{v}, \mathrm{s}_{1}, \mathrm{~s}_{2}, \mathrm{~s}_{3}, \mathrm{n}_{2}, \mathrm{n}_{3} \in \mathrm{W}$ and $\alpha \in \Gamma$

Hence by (ii), gives the result .

\section{Theorem 2.4 :}

Assume that $\mathrm{W}$ is a 2-torsion free semiprime $\Gamma$-near-ring and $\mathrm{P}, \mathrm{Q}$ are two generalized 3derivations of $\mathrm{W}$ with associated 3-

derivations $p$ and $q$ of $W$ respectively. If $P$ and $q$ are perpendicular and $Q$ and $p$ are perpendicular, then we have

(i) $\mathrm{p}\left(\mathrm{q}\left(\mathrm{s}_{1}, \mathrm{~s}_{2}, \mathrm{~s}_{3}\right), \mathrm{n}_{2}, \mathrm{n}_{3}\right)=0$ and

$\mathrm{P}\left(\mathrm{Q}\left(\mathrm{s}_{1} \alpha \mathrm{s}_{1}^{\prime}, \mathrm{s}_{2}, \mathrm{~s}_{3}\right), \mathrm{n}_{2}, \mathrm{n}_{3}\right)=\mathrm{P}\left(\mathrm{Q}\left(\mathrm{s}_{1}, \mathrm{~s}_{2}, \mathrm{~s}_{3}\right)\right.$, $\left.\mathrm{n}_{2}, \mathrm{n}_{3}\right) \alpha \mathrm{s}_{1}^{\prime}$

for all $\mathrm{s}_{1}, \mathrm{~s}_{1}^{\prime}, \mathrm{s}_{2}, \mathrm{~s}_{3}, \mathrm{n}_{2}, \mathrm{n}_{3} \in \mathrm{W}$ and $\alpha \in \Gamma$.

(ii) $\mathrm{q}\left(\mathrm{p}\left(\mathrm{s}_{1}, \mathrm{~s}_{2}, \mathrm{~s}_{3}\right), \mathrm{n}_{2}, \mathrm{n}_{3}\right)=0$ and

$\mathrm{Q}\left(\mathrm{P}\left(\mathrm{s}_{1} \alpha \mathrm{s}_{1}^{\prime}, \mathrm{s}_{2}, \mathrm{~s}_{3}\right), \mathrm{n}_{2}, \mathrm{n}_{3}\right)=\mathrm{Q}\left(\mathrm{P}\left(\mathrm{s}_{1}, \mathrm{~s}_{2}, \mathrm{~s}_{3}\right)\right.$, $\left.\mathrm{n}_{2}, \mathrm{n}_{3}\right) \alpha \mathrm{s}_{1}^{\prime}$

for all $\mathrm{s}_{1}, \mathrm{~s}_{1}^{\prime}, \mathrm{s}_{2}, \mathrm{~s}_{3}, \mathrm{n}_{2}, \mathrm{n}_{3} \in \mathrm{W}, \alpha \in \Gamma$. 


\section{Proof :}

(i)since $\mathrm{P}$ and $\mathrm{q}$ are perpendicular, we have $\mathrm{P}\left(\mathrm{s}_{1}, \mathrm{~s}_{2}, \mathrm{~s}_{3}\right) \alpha \mathrm{s} \beta \mathrm{q}\left(\mathrm{n}_{1}, \mathrm{n}_{2}, \mathrm{n}_{3}\right)=0$ for all $\mathrm{s}, \mathrm{s}_{1}, \mathrm{~s}_{2}, \mathrm{~s}_{3}, \mathrm{n}_{1}, \mathrm{n}_{2}, \mathrm{n}_{3} \in \mathrm{W}$ and $\alpha, \beta$ $\in \Gamma$.

Replacing $\mathrm{s}_{1}$ by $\mathrm{s}_{1}{ }^{\prime} \delta \mathrm{s}_{1}$ in above equation, by Lemma 2.2 , we have $0=\mathrm{P}\left(\mathrm{s}_{1}^{\prime} \delta \mathrm{s}_{1}, \mathrm{~s}_{2}, \mathrm{~s}_{3}\right) \alpha \mathrm{s} \beta \mathrm{q}\left(\mathrm{n}_{1}, \mathrm{n}_{2}, \mathrm{n}_{3}\right)$ $=\mathrm{P}\left(\mathrm{s}_{1}{ }^{\prime}, \mathrm{s}_{2}, \mathrm{~s}_{3}\right) \delta \mathrm{s}_{1} \alpha \mathrm{s} \beta \mathrm{q}\left(\mathrm{n}_{1}, \mathrm{n}_{2}, \mathrm{n}_{3}\right)$ $+\mathrm{s}_{1}^{\prime} \delta \mathrm{p}\left(\mathrm{s}_{1}, \mathrm{~s}_{2}, \mathrm{~s}_{3}\right) \alpha \mathrm{s} \beta \mathrm{q}\left(\mathrm{n}_{1}, \mathrm{n}_{2}, \mathrm{n}_{3}\right)$ $=\mathrm{s}_{1}^{\prime} \delta \mathrm{p}\left(\mathrm{s}_{1}, \mathrm{~s}_{2}, \mathrm{~s}_{3}\right) \alpha \mathrm{s} \beta \mathrm{q}\left(\mathrm{n}_{1}, \mathrm{n}_{2}, \mathrm{n}_{3}\right)$ for all $\mathrm{s}, \mathrm{s}_{1}, \mathrm{~s}_{1}^{\prime}, \mathrm{s}_{2}, \mathrm{~s}_{3}, \mathrm{n}_{1}, \mathrm{n}_{2}, \mathrm{n}_{3} \in \mathrm{W}, \alpha$, $\beta, \delta \in \Gamma$.

Semiprimeness of $\mathrm{W}$ yields that $\mathrm{p}\left(\mathrm{s}_{1}, \mathrm{~s}_{2}, \mathrm{~s}_{3}\right) \alpha \mathrm{s} \beta \mathrm{q}\left(\mathrm{n}_{1}, \mathrm{n}_{2}, \mathrm{n}_{3}\right)=0$ for every $\mathrm{s}, \mathrm{s}_{1}, \mathrm{~s}_{2}, \mathrm{~s}_{3}, \mathrm{n}_{1}, \mathrm{n}_{2}, \mathrm{n}_{3} \in \mathrm{W}, \alpha, \beta \in$ $\Gamma$.

Thus $\mathrm{p}$ and $\mathrm{q}$ are perpendicular

By Theorem 2.3, we have

$\mathrm{p}\left(\mathrm{q}\left(\mathrm{s}_{1}, \mathrm{~s}_{2}, \mathrm{~s}_{3}\right), \mathrm{n}_{2}, \mathrm{n}_{3}\right)=0$

for every $\mathrm{s}_{1}, \mathrm{~s}_{2}, \mathrm{~s}_{3}, \mathrm{n}_{2}, \mathrm{n}_{3} \in \mathrm{W}$. (2.4)

Since $\mathrm{P}$ and $\mathrm{q}$ are perpendicular and $\mathrm{Q}$ and $\mathrm{p}$ are perpendicular, we have

$\mathrm{P}\left(\mathrm{s}_{1}, \mathrm{~s}_{2}, \mathrm{~s}_{3}\right) \alpha \mathrm{q}\left(\mathrm{n}_{1}, \mathrm{n}_{2}, \mathrm{n}_{3}\right)=0$

and

$\mathrm{Q}\left(\mathrm{s}_{1}, \mathrm{~s}_{2}, \mathrm{~s}_{3}\right) \alpha \mathrm{p}\left(\mathrm{n}_{1}, \mathrm{n}_{2}, \mathrm{n}_{3}\right)=0$

for every $\mathrm{s}_{1}, \mathrm{~s}_{2}, \mathrm{~s}_{3}, \mathrm{n}_{1}, \mathrm{n}_{2}, \mathrm{n}_{3} \in \mathrm{W}, \alpha \in \Gamma$.

So we get

$\mathrm{P}\left(\mathrm{Q}\left(\mathrm{s}_{1} \alpha \mathrm{s}_{1}^{\prime}, \mathrm{s}_{2}, \mathrm{~s}_{3}\right), \mathrm{n}_{2}, \mathrm{n}_{3}\right)$

$=\mathrm{P}\left(\mathrm{Q}\left(\mathrm{s}_{1}, \mathrm{~s}_{2}, \mathrm{~s}_{3}\right) \alpha \mathrm{s}_{1}{ }^{\prime}+\mathrm{s}_{1} \alpha \mathrm{q}\left(\mathrm{s}_{1}{ }^{\prime}, \mathrm{s}_{2}, \mathrm{~s}_{3}\right), \mathrm{n}_{2}\right.$, $\left.\mathrm{n}_{3}\right)$

$=\mathrm{P}\left(\mathrm{Q}\left(\mathrm{s}_{1}, \mathrm{~s}_{2}, \mathrm{~s}_{3}\right) \alpha \mathrm{s}_{1}^{\prime}, \mathrm{n}_{2}, \mathrm{n}_{3}\right)+\mathrm{P}\left(\mathrm{s}_{1} \alpha \mathrm{q}\left(\mathrm{s}_{1}^{\prime}\right.\right.$

$\left.\left., \mathrm{s}_{2}, \mathrm{~s}_{3}\right), \mathrm{n}_{2}, \mathrm{n}_{3}\right)$

$=\mathrm{P}\left(\mathrm{Q}\left(\mathrm{s}_{1}, \mathrm{~s}_{2}, \mathrm{~s}_{3}\right), \mathrm{n}_{2}, \mathrm{n}_{3}\right) \alpha \mathrm{s}_{1}^{\prime}+\mathrm{Q}\left(\mathrm{s}_{1}, \mathrm{~s}_{2}, \mathrm{~s}_{3}\right.$

) $\alpha \mathrm{p}\left(\mathrm{s}_{1}^{\prime}, \mathrm{n}_{2}, \mathrm{n}_{3}\right)+$

$\mathrm{P}\left(\mathrm{s}_{1}, \mathrm{n}_{2}, \mathrm{n}_{3}\right) \alpha \mathrm{q}\left(\mathrm{s}_{1}^{\prime}, \mathrm{s}_{2}, \mathrm{~s}_{3}\right)+\mathrm{s}_{1} \alpha \mathrm{p}\left(\mathrm{q}\left(\mathrm{s}_{1}^{\prime}, \mathrm{s}_{2}\right.\right.$, $\left.\left.\mathrm{s}_{3}\right), \mathrm{n}_{2}, \mathrm{n}_{3}\right)$

By using relation (2.4), (2.5) and (2.6) in the last equation, we get

$\mathrm{P}\left(\mathrm{Q}\left(\mathrm{s}_{1} \alpha \mathrm{s}_{1}^{\prime}, \mathrm{s}_{2}, \mathrm{~s}_{3}\right), \mathrm{n}_{2}, \mathrm{n}_{3}\right)=\mathrm{P}\left(\mathrm{Q}\left(\mathrm{s}_{1}, \mathrm{~s}_{2}, \mathrm{~s}_{3}\right)\right.$, $\left.\mathrm{n}_{2}, \mathrm{n}_{3}\right) \alpha \mathrm{s}_{1}^{\prime}$

for all $\mathrm{s}_{1}, \mathrm{~s}_{1}^{\prime}, \mathrm{s}_{2}, \mathrm{~s}_{3}, \mathrm{n}_{2}, \mathrm{n}_{3} \in \mathrm{W}, \alpha \in \Gamma$.

(ii)The proof is the same way to the proof of

(i)

\section{References}

[1] E. F. Adhab , 2016 . n-Additive Mappings in Prime and Semiprime Near-Rings, PHD.

Thesis, Baghdad University, Iraq . [2] I. N. Herstein , 1969 . Topics in Ring Theory, University of Chicago Press, Chicago .

[3] K.K. Dey , A. C. paul and I. S. Rakhimov , 2012 . On prime gamma-near-rings with generalized derivations, International Journal of Math. and Math. Sci. , doi:

10.1155/2012/625968.

[4] K. K. Dey , A. C. Paul and I. S. Rakhimov, 2012 . Orthogonal Generalized Derivations in Semiprime Gamma Near-Rings , International Journal of Algebra, 6(23) : 1127-1134 .

[5] W. E. Barnes, 1966 . On the $\Gamma$-rings of Nabusawa ,Pacific J. Math., 18 : 411-422 . 
على الحلقات المقتربة كامـا شبه الاولية مع الاثثقاقات - 3 المعممة المتعامدة

$$
\text { قاكم العلوم التطد سعيدة التبية }
$$

في هذا البحث قدمنا مفهوم الاشتقاقات - 3 المعممة المتعامدة في الحلقات المقتربة كاما شبه الاولية وقدمنا العديد من الثروط الكافية و الضرورية للاشتقاقات - 3 المعممة على الحقات المقتربة كاما شبه الاولية لتصبح متعامدة .

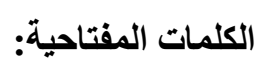

الحلقة المقتربة كاما شبه الاولية ، الاشتقاقات -3 ، الاشتقاقات -3 المعممة ، الاشتقاقات -3 المتعامدة ، الاشتقاقات -3 المعممة المتعامدة . 